\title{
CAPSULE COMMENTARIES \\ Capsule Commentary on Klein et al., Categorical Risk Perception Drives Variability in Antibiotic Prescribing in the Emergency Department: a Mixed Methods Observational Study
}

\author{
Jeffrey A. Linder, MD, MPH, FACP \\ Division of General Internal Medicine and Geriatrics, Northwestern University Feinberg School of Medicine, Chicago, IL, USA.
}

J Gen Intern Med 32(10): 1130

DOI: $10.1007 / \mathrm{s} 11606-017-4136-5$

(C) Society of General Internal Medicine 2017

$\mathrm{K}$ lein and colleagues compared emergency department clinicians' acute respiratory infection (ARI) antibiotic prescribing with clinicians' decision-making frameworks, or "gists," regarding ARIs and antibiotics. ${ }^{1}$ Clinicians who had survey responses that aligned with what they describe as a "why-not-take-a-risk" gist - probably better described as the “antibiotics-are-low-risk-and-the-patient-won't-get-betterwithout-them" gist — had higher antibiotic prescribing. Clinicians who had survey responses that aligned with an "antibiotics-may-be-harmful" gist had lower antibiotic prescribing.

These results are an interesting foray into the association between decision-making mental constructs and prescribing behavior. However, there remains much to understand and work to be done. The patient, clinical, and clinician factors along with the two gists examined in this study do not tell the whole story. Contextual, communication, and social factors have a role. ${ }^{2-5}$

The importance of the association between the gists and antibiotic prescribing, because of low prevalence and weak strength of association, could be small relative to other factors. For example, in the main model, the "why-not-take-a-risk" gist was associated with $28 \%$ increased odds of prescribing antibiotics, which only increases absolute antibiotic prescribing from, for example, $64 \%$ to $69 \%$.

Diagnoses were the most powerful predictors of antibiotic prescribing, but future studies need more diagnostic clarity. The diagnostic groupings in this study conflated diagnoses that never, sometimes, or always require antibiotics (e.g., the common cold, sinusitis, and tonsillitis are in the same category). More importantly, individual clinicians could be working from completely different gists depending on the diagnosis.
For example, the same clinician might have very different mental constructs - and probably should - whether they think they are treating a patient with a non-specific upper respiratory tract infection, pharyngitis, acute bronchitis, or pneumonia.

With more understanding and diagnostic clarity, future interventions might attempt "gist shift" and see if behavior changes. For ARIs, interventions could emphasize the harms of antibiotics for individual patients - especially the idea that patients could be qualitatively worse for having taken an antibiotic. Beyond ARIs, other areas where it seems important to understand gists include opioid prescribing (perception of suffering vs. drug risks), preventive services (benefits vs. inconvenience), and chronic disease management (urgency vs. competing priorities).

Corresponding Author: Jeffrey A. Linder, MD, MPH, FACP; Division of General Internal Medicine and GeriatricsNorthwestern University Feinberg School of Medicine, Chicago, IL 60611, USA (e-mail: jlinder@northwestern.edu).

\section{Compliance with Ethical Standards:}

Conflict of Interest: The author has no conflicts of interest with this article.

\section{REFERENCES}

1. Klein EY, Martinez EM, May L, Saheed M, Reyna V, Broniatowski DA. Categorical risk perception drives variability in antibiotic prescribing in the emergency department: a mixed methods study. J Gen Intern Med. 2017. doi:10.1007/s11606-017-4099-6.

2. Meeker D, Linder JA, Fox CR, et al. Effect of behavioral interventions on inappropriate antibiotic prescribing among primary care practices: a randomized clinical trial. JAMA. 2016;315(6):562-570.

3. Pineros D, Doctor JN, Friedberg MW, Meeker D, Linder JA. Cognitive reflection and antibiotic prescribing for acute respiratory infections. Fam Pract. 2016;33(3):309-311

4. Tannenbaum D, Doctor JN, Persell SD, et al. Nudging physician prescription decisions by partitioning the order set: results of a vignettebased study. J Gen Intern Med. 2015;30(3):298-304.

5. Linder JA, Doctor JN, Friedberg MW, et al. Time of day and the decision to prescribe antibiotics. JAMA Intern Med. 2014;174(12):2029-2031. 